\title{
The Influence of Marvel in the Chinese Teenagers
}

\author{
Hengda Zhu ${ }^{1, \mathrm{a}, \uparrow}$, Yishu Ren ${ }^{2, \mathrm{~b}, \uparrow}$, Shuhui Chen ${ }^{3, \mathrm{c}, \uparrow}$ \\ ${ }^{1}$ College of Communication and Art Design, University of Shanghai for Science and Technology, Shanghai, China \\ ${ }^{2}$ Dulwich International School Zhuhai, Zhuhai, China \\ ${ }^{3}$ Shanghai Experimental Cambridge School, Shanghai, China \\ *Corresponding author.Email: ${ }^{a} 1820550630 @$ st.usst.edu.cn, ${ }^{b} 22 R e n Y 1 @ d u l w i c h-z h u h a i . c n,{ }^{c} 2972214203 @ q q . c o m$ \\ These authors contributed equally.
}

\begin{abstract}
This research focus on the acceptance of Marvel films among young Chinese audiences, and the application of Marvel films in related fields. Unlike other papers, researchers get the results from young Chinese audiences. Many people are more interested in comic books or spin-offs after watching a Marvel movie. It cannot be ignored that special effect technology is the fundamental basis to present a stunning fighting scene or spectacular fictional worlds, especially for the youth. This paper also discusses research methods and results. The methodology is the questionnaire survey and the result analysis, to directly get fresh first-hand data from young people and connect it with the concept of The Times. As has been discussed above, the continuity of twilight films may prove its popularity. The audience's affection for Marvel comes from the time bias of the series. From the content point of view, researchers should notice the characterisation in the Marvel Movie Universe movies. All in all, we can generate several instructions from the example of MCU movies. Those may not only helpful to Marvel but also can be used for reference to the Chinese movie industry. Especially nowadays there are more and more movies adapted from Chinese original comics. They are also mainly designed around a similar theme, the story of Chinese gods. This similarity between those may prove the reliability and practicability of these instructions.
\end{abstract}

Keywords: Marvel, Spread, film, Chinese Youth

\section{INTRODUCTION}

Marvel Studios is a famous American company producing a series of hero films. In 1939, Timely Comic Company was established and changed the name to Marvel in 1961. The company owned Iron Man, SpiderMan, Captain America, Thor, The Hulk, Black Widow, Wolverine that about 8,000 superheroes and The Avengers, Fantastic Four, X-man, Guardians of the Galaxy and so on superhero teams.

In the 1980s, with the great improvement of technology, comics already could not be satisfied by the public so that leading to a recession of Marvel. In this case, Marvel sold out all rights of adaptation to Merrill Lynch to raise funds. Luckily, the desperate gamble won-Iron Man brought 5.85 billion US dollars boxoffice sweeping the world with 1.8 billion dollars in 2008 which kicked off a "Golden Age" of Marvel. After that, Walt Disney bought Marvel and more than 5,000 IP for 4.4 billion, making Marvel IP resources more complete. [1]
Marvel films created a concept of the Marvel Cinematic Universe (abbreviation: MCU) and threephases strategy. According to the official data of Marvel, Marvel film has developed to the fourth phase and the temporal span is more than ten years (2008-2020). The screening of the first film, Iron Man, in 2008 has marked the beginning of the first phase of creation which is also named the gathering of the Avengers. In this stage, new superheroes have been introduced to pave the way for MCU. It has been ended in 2012 with The Avengers I; Iron Man III has been released in 2013 as the beginning of phase two - the opening of a new world and has been ended by Ant-Man in 2015. It mainly handles the fallout from The Avengers; 2016's Captain America: Civil War has opened the phase three: Rise of the Unknown, leading up to 2019's Spider-Man: Far From Home. [2] The course of Marvel films is clear and definite so that brings the audience to MCU step by step.

Talking about the box office performance of Marvel films in China. According to online data, Spider-Man: Far from Home has now grossed 1.026 billion yuan on 
the Chinese mainland, bringing the total box office of the Marvel Universe -- all 23 films so far -- to more than 20 billion yuan. The top five $M C U$ films in China are Avengers 4, Avengers 3, Avengers 2, Captain America 3, and Captain Marvel, respectively. Marvel Cinematic Universe hits 10 billion yuan on the Chinese mainland when its 18th film, Black Panther, and the second 10 billion yuan was much faster, unlocking 20 billion yuan in just five films. The 20 billion yuan accounted for 5.88 percent of the total box office in China's film market since 2008. This can also be indirect proof of how quickly the development of China's film market is.

\section{LITERATURE REVIEW}

According to the research on CNKI, researchers have reviewed the previous studies about Marvel industry. From a broad view, most of them focus on the industry development and which kind of culture it wants to convey that compares to other ideological films_— such as DC film. But none of the research paper refers to the media transmission of Marvel films. Then researchers keep searching five more articles that have a strong relationship with this research question.

Zhao Hui has briefly analysed "audience's feeling" and "group influence" to support why Marvel caused a sensation [3]. "The Narrative Strategy and Cultural Communication of Adapted Films" written by He Ming has focused on how Marvel was promoted to spread from the perspectives of individualism and universalism [4]. And then, Xie Linshan has emphasised more detail on the theoretical knowledge of the text [5]. How does the text attract the audience as the core of the film? Another study is written by $\mathrm{Hu}$ Yiqiu who has explored the special role of the Marvel fan culture in its cross-cultural communication [6]. This is also one of the few articles to analyse - the impact of communication from the audience's perspective. After that, Liu Guohao in his article "A study of the Communication Effects of Marvel Superhero films in China" has discussed the reason why Marvel is popular in China [7]. Liu Guohao's article is the one mostly related to this topic as both of the two use questionary to collect data. The difference is that the writer has thought responders between 18 to 30 is one of the limitations but our main responder is it. Therefore, it can be seen that, there is a short paper from this research doing Marvel media influence on teenagers and young adults. Moreover, this perspective is important as China is the biggest film market in the world. Chinese audience help to boost the success of Marvel. Therefore, the research gap is to sum up the experience of the development of Marvel. After this study, some useful conclusions can be given for Chinese films since it is just getting started. From the perspective of the narrative characteristics of Marvel superhero movies, the superhero protagonists under Marvel have the characteristics of anti-hero images, and their growth and transformation often rely on external events to promote [8]. From a cross-cultural point of view, Marvel movies emphasize concise story pastors and low-context language styles [9]. From the perspective of the brand, Marvel movies pay attention to the integration of IP resources and strengthen the brand image through the cooperation of IP [10].

\section{METHODOLOGY}

In this study, qualitative and quantitative analysis will be carried out by means of a questionnaire survey to investigate the dissemination of Marvel movies among young audiences, so as to study why Marvel has high popularity in China.

Choosing the questionnaire to collect information has many advantages, such as high efficiency, objectivity, uniform and universality. Most of the questions are answered by people around us, so Researchers can know more about people around us from the results. Our group collected information by means of a questionnaire survey and made it by using the questionnaire star platform. Researchers have released the questionnaire on WeChat Moments and Groups, etc. We have delivered the questionnaire on March 10 and finished the questionnaire on March 12. Researchers have asked a total of 10 questions and received 166 sets of answers, with the valid questionnaire accounting for $83.3 \%$.

However, the questionnaire survey still has some limitations, for example, the scope is not wide enough, Researchers can only know the situation of the people around us, rather than the situation of the whole of China, and some of the respondents may omit information, omit to fill and so on.

In the recovered data, the proportion of male and female applicants is 5 to 4 . Most applicants are aged 1618 , accounting for $54.22 \%$, and 37.35 percent are aged $19-22$ and $8.43 \%$ are above 22 years old. Between 2008 and 2018, Marvel had the highest number of contacts, with $73.49 \%$. Most of them have learned about Marvel through promotional films and recommendations from close friends. Eighty-three percent of people watch Marvel in the theatre. 27.71 percent have watched 10-18 movies, followed by $1-3$ at 25.3 percent. Fifty-six percent of people said they would continue to follow relevant movies, and 84 percent of people gave favourable comments on Marvel movies. The highest proportion of people who follow Marvel movies is neither too much nor too little. In terms of whether they would recommend Marvel or China to people around them, the overall score of Chinese Marvel is 3.35, while Marvel is 3.51.

\section{DISCUSSION}

In the question "How many Marvel films have you seen?",4.71 percent of people have not seen a Marvel 
film. 24.71 percent have seen only $1-3$ of them; 18.82 percent have seen $4-9 ; 28.24$ percent have seen 10-18; and 23.63 percent have seen all. These data can show that Marvel is very popular in youth group of China.

In the question "When did you start paying attention to Marvel?", the results show that before 2008, when the first MCU film Iron Man has been released, only 12.94 percent of people has been exposed to Marvel. Between 2008 and 2012, 24.71 percent of people pay attention to Marvel films. During this time, MCU's first-stage films, such as Captain America, The Mighty Thor, and the Avengers, has been released. Between 2013 and 2015, the second phase of the MCU, 22.35 percent of people start to come into contact. A further 12.94 percent of people will not be in contact until 2018. With all the statics above, there has been a clear growing tendency that people become interested in Marvel films. It can be contributed to the success of MCU's three-phases strategy, for its developing storylines continually attract people's attention. Thus, this series will keep "on trending" in social medias, people's daily talks and other fields in daily life, which may enhance the effect of interpersonal communication to a large extent, especially in the youth groups.

When it comes to "How many people around you pay attention to Marvel films, comics, spin-offs?", the survey shows that only $5.88 \%$ think there are few people around them who are interested in Marvel and $7.06 \%$ think there are still a few people focusing on Marvel although not very much. There is $42.35 \%$, which are accounted for the largest proportions, describe "no more, no less" as the amount of those who are interested in Marvel around them. Besides, $31.76 \%$ and $12.94 \%$ of people think there are many and sight of fans of Marvel. This can be another proof that Marvel does spread widely among youth group in China.

When interviewees are asked will you be more interested in comic books or spin-offs after watching a Marvel film, as can be seen from the figure, over $50 \%$ of interviewees will show more interests in Marvel. Among them, nearly 60 percent of male interviewees would begin to follow with interest MCU's relevant comic books or TV series, while only 35 percent of female interviewees would be attracted to pay attention to the MCU's extra production. It does not accord with the Chinese present situation of comics and animation audiences, which is expected to be nearly the same. So, it demonstrates that sexual distinction plays an important role in the communication effects of MCU films, especially in retaining their audiences.

Therefore, combined with the previous two questions, it can be concluded that the MCU is indeed a widespread appeal to Chinese youth audiences, not only for its films' market but also its derivative works. However, its effects may be diverse in genders.
When talking about through which channel they know about MCU films, according to the information obtained, most people, both men and women, learn about MCU films through film promotions $(69.41 \%)$ and recommendation from friends $(61.18 \%)$, while a small number $(23.53 \%)$ of people learn about it through wemedia productions such as some mush-up videos or articles. Based on the previous results, it may conclude that Marvel has received great success in popularizing itself in China youth groups. From this question, not only did the three-phases strategy that sparks continuous public discussion take effect but also its creative trailers and other promotional channels play an important role.

To find out the c2c effects of MCU films, this research also set the question that whether people would recommend Marvel films to people around them. Interviewees will rate how much they would like to do so. The average score of male students is 3.23 , while that of female students is 3.48 , indicating that female students are more willing to recommend it. In terms of this outcome, it may seem a little contradictory to the conclusion above, because the communication effects are better in the female group, while they do not want to pay attention to derivative works. However, the content of films themselves may attribute this phenomenon. As a superhero film, it may put its focus on the scene of the fights and demonstrations of superheroes' superpowers, instead of love scenes. So, they may become interested in these spectacular series films but no more. The fan art may seem more attractive to them.

This research has also studied that through what channels do the youth watch Marvel films. It turns out that 83.53 percent chose to go to the cinema and 68.24 percent chose to watch it through online media as well. According to this question, it can be found that people are more willing to go to the cinema especially for this kind of blockbuster mentality. However, the growing tendency of stream media platform also cannot be ignored.

Furthermore, this research also inquires the reasons people like Marvel films. In their responses, more than forty people have mentioned "plot" and "universe", and more than twenty people have mentioned effects or visual effects and some things like that. "hero" and "role" rank the third place. Some other words like "production" and "superpower" are also mentioned several times. It reveals that the youth still regard the narrative of the film as a fundamental point although the visual effects are more and more important and popular nowadays.

The ones who dislike Marvel are also included. Although some people are fond of this kind of huge narrative system, some others do not have this interest. There are $15.29 \%$ people expressing that they have no appetite for MCU films due to the complicated storylines. Thus, the huge narrative system seems to be a risk as an asset for series films. In terms of the gender proportion in this questionnaire samples, males take up $55.29 \%$. When 
it comes to age-cohorts, 16-18 years old group contributes the largest proportion $(55.47 \%), 19-22$ years old group takes the second. So, this research may provide quite referential advices for films that will be shown in China whose target is youth people.

All in all, researchers can generate several instructions from the example of MCU films. Those may not only be helpful to Marvel but also can be used for reference to the Chinese film industry. Especially nowadays there are more and more films adapted from Chinese original comics. They are also mainly designed around a similar theme, the story of Chinese gods. This similarity between those may prove the reliability and practicability of these instructions.

First, as has been discussed above, the continuity of series films may prove its popularity. The audience's affection for Marvel comes from the time bias of the series. Because of the long-time span of the series, the Marvel Cinematic Universe has achieved control over longitudinal time. From Iron Man 1 in 2008 to The Avengers 4 in 2019, 22 films in 11 years, Marvel Pictures has ushered in this magnificent Marvel universe through temporal spans and has already ushered in. More importantly, 22 films are released around the world, and a younger generation of viewers have watched the characters going from weak to powerful and eventually superheroes. "The Avengers" in the film continues to grow, and so do the audience outside the film. They grow up step by step, graduation, going into society. Those 11 years of many of their youth memories have been accompanied by a long time, forming a special film feeling. It is this kind of feeling that makes MCU bundled with those youth. Also, the continuous discussions around oneself deepens the audience's impression of the film.

Secondly, from the content point of view, we should notice the characterization in the MCU films. Although the characters are superheroes, we can still find some common sensation or even weaknesses from them, such as spiderman's tenderness, Iron Man's pride, Hulk's grumpy temper and so on, instead of a perfect image. This will provide immersion for the audience to satisfy their longing for justice and spur themselves on. It means that the storyline should be connected to the real social problem or some phenomena are closely watched, so that the young generation may let themselves into the film and sense it from the bottom of their hearts.

Finally, it cannot be ignored that special effect technology is the fundamental basis to present a stunning fighting scene or spectacular fictional worlds, especially for the youth. It is because that fantasizing about space is also a way for young people to construct the psychological and spiritual world.

\section{CONCLUSION}

The present study was designed to determine the effect of the Marvel movie on teenagers and young adults in China. This study contributes to our standing of how foreign films are opening up in the Chinese markethow they attract audiences, in this case, Chinese teenagers. Also, the finding from this study makes several contributions to the current literature. First, fill the research gap in the teenager age group as they take a large proportion of crowds of cinema goers. Secondly, it explores the subsequent effects of derivative products outside the movies on the audience — whether they are long-term effects. However, this study was limited by the absence of responders which only 166 whereas reflected the whole Chinese teenagers and young adults is not enough. Even though the sample is not as widely as we expected, but the message that conveys to us is still useful. The exploration of Marvel movies can inspire the Chinese animation because Chinese animation is now on the way to develop its own universe and try to make a series of movies like MCU. And both of the two are described and satisfied the hero complex in the young people's hearts. So, Marvel's development can be used as a model.

\section{REFERENCES}

[1] Y, Gao, A study of Superhero's in the Universe Series of Marvel Cinematic, 2019-3-5, P9.

[2] Y.Z Chen, Marvel Cinema Studies, 2017-3-29, P12.

[3] H. Zhao, Analysis of the sensational phenomenon caused by series films from the perspective of communication bias, Audio and visual interpretation, 2021, 1, P79.

[4] M. He, The Narrative Strategy and Cultural Communication of Adapted Films, Academic BBS, 2019-12, 729, P30.

[5] L.S Xie, A brief analysis of the intercultural communication of Marvel superhero movies, The Silk Road, 2017-8, (355), P11.

[6] Y.Q Hu, A Study on the Cross-Cultural Communication and Fan Culture of Marvel Movie Series, 2020-3-20.

[7] G.H Liu, A study of the Communication Effects of Marvel Superhero Movies in China, 2020-5.

[8] Y.H. Zhao, Narrative Mode and Characteristics of American Superhero Films in the New Century -- A Case Study of Marvel and DC Superhero Films, Audio-Visual 2021, (02),55-58.

[9] J.R. QI, Intercultural Elements and Defamiliarization of Marvel Movies Intensive Reading, News Communications 2021, (01),9-11.

[10] X.H. Wang, Z.H. Qin, Marvel Brand IP Image Building and Communication Intensive Reading, International Brand Watch 2020, (29),73-75. 